University of Wollongong

Research Online

Faculty of Informatics - Papers (Archive)

Faculty of Engineering and Information

Sciences

27-8-2007

\title{
Complementary Channel Estimation and Synchronization for OFDM
}

\author{
Darryn Lowe \\ University of Wollongong, darrynl@uow.edu.au \\ Xiaojing Huang \\ University of Wollongong, huang@uow.edu.au
}

Follow this and additional works at: https://ro.uow.edu.au/infopapers

Part of the Physical Sciences and Mathematics Commons

\section{Recommended Citation}

Lowe, Darryn and Huang, Xiaojing: Complementary Channel Estimation and Synchronization for OFDM 2007.

https://ro.uow.edu.au/infopapers/621

Research Online is the open access institutional repository for the University of Wollongong. For further information contact the UOW Library: research-pubs@uow.edu.au 


\title{
Complementary Channel Estimation and Synchronization for OFDM
}

\author{
Abstract \\ In this paper, we present a new type of packet preamble for orthogonal frequency division multiplexing \\ (OFDM) systems that is based on a complementary sequence pair. It is shown how this approach permits \\ a receiver to dynamically choose between frequency-domain equalization (FDE) and timedomain \\ equalization (TDE). With FDE offering a low-complexity zero-forcing (ZF) equalization and TDE facilitating \\ easy minimum mean square error (MMSE) estimation, it is concluded that a spectrally-flattened \\ complementary sequence pair offers an outstanding combination of flexibility and performance. \\ Disciplines \\ Physical Sciences and Mathematics

\section{Publication Details} \\ This conference paper was originally published as Lowe, D, Huang, $X$, Complementary Channel Estimation \\ and Synchronization for OFDM, 2nd International Wireless Broadband and Ultra Wideband \\ Communications Auswireless 2007, 27-30 Aug, 23-23.
}




\title{
Complementary Channel Estimation and Synchronization for OFDM
}

\author{
Darryn Lowe and Xiaojing Huang \\ School of Electrical, Computer and Telecommunications Engineering \\ University of Wollongong \\ Wollongong, Australia, 2522 \\ Email: \{darrynl, huang\}@uow.edu.au
}

\begin{abstract}
In this paper, we present a new type of packet preamble for orthogonal frequency division multiplexing (OFDM) systems that is based on a complementary sequence pair. It is shown how this approach permits a receiver to dynamically choose between frequency-domain equalization (FDE) and timedomain equalization (TDE). With FDE offering a low-complexity zero-forcing (ZF) equalization and TDE facilitating easy minimum mean square error (MMSE) estimation, it is concluded that a spectrally-flattened complementary sequence pair offers an outstanding combination of flexibility and performance.
\end{abstract}

\section{INTRODUCTION}

Coherent signalling over frequency-selective channels requires that the receiver estimate and equalize the channel prior to demodulation. To that end, one of the most significant advantages of orthogonal frequency division multiplexing (OFDM) systems [1], typified by the block diagram of Fig. 1 , is that the highlighted channel estimation and equalization stages are both conceptually and computationally simple. Since this allows an OFDM transceiver to be low-power and low-cost, OFDM systems are becoming increasingly popular in applications ranging from wireless personal area networks (WPANs) to digital television.

The simplest OFDM channel estimation is a zero-forcing (ZF) approximation of $N$ complex coefficients that rotate and scale each of the $N$ subcarriers. This estimation is commonly achieved using an explicit preamble training sequence known to both sender and receiver. Although blind estimation [2] or differential modulation alternatives exist, they suffer from either longer convergence times and/or degraded performance.

Most OFDM channel estimation sequences (CES) possess a flat power-spectral density (PSD) so that each subcarrier can be estimated to the same accuracy. For example, in multiband OFDM (MB-OFDM) [3], the CES is obtained by taking the inverse fast fourier transform (IFFT) of $N$ random equalmagnitude quadrature phase shift keying (QPSK) constellations. This allows the receiver to perform a ZF frequencydomain estimation (FDE) at the cost of little more than a single fast fourier transform (FFT).

The problem with a ZF FDE is that each subcarrier is estimated in isolation. Since the maximum delay spread of the channel is, by design, only a fraction of the duration of the OFDM symbol, the mean square error (MSE) of such an estimate will be increased. In order to better exploit

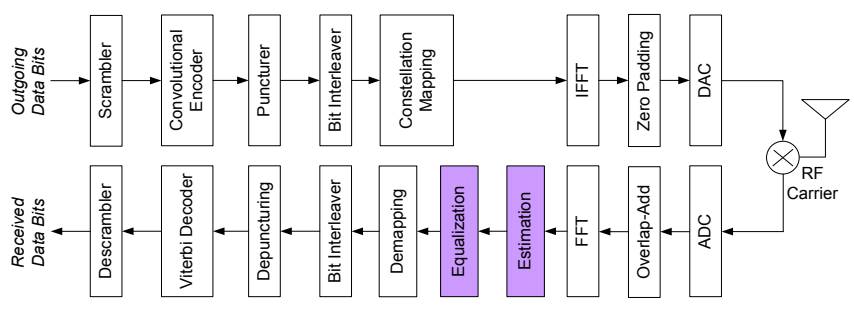

Fig. 1. Block diagram of an generic OFDM transmitter.

subcarrier inter-correlation, a minimum mean square error (MMSE) estimation can be used [4] to improve performance. Unfortunately, in addition to requiring explicit knowledge of the channel power delay profile, an MMSE estimation is computational expensive since an original ZF FDE estimate must be convolved by a potentially large smoothing filter.

Regardless of the sophistication of receiver processing, the design of a CES suitable for FDE is straight-forward. Although the number of candidate sequences is very large, with even simple QPSK symbol constellations yielding $4^{N}$ candidate sequences, all such sequences are equivalent in terms of channel estimation capability. Accordingly, the preferred sequence is usually selected using secondary criteria such as minimizing the peak-to-average power ratio (PAPR).

Another approach to channel estimation is to perform a time-domain estimation (TDE) that uses a a CES with an ideally impulse auto-correlation function (ACF). In this case, since the estimate is of the time-domain channel impulse response (CIR), an FFT is still required to transform the CIR such that it can be used with a conventional OFDM one-tap equalizer.

The advantage of TDE over FDE is that MMSE smoothing can be applied at much lower complexity since the smoothing filter convolution that was prohibitively expensive for FDE is reduced to to multiplication by virtue of the fast-convolution property of Fourier transforms. Unfortunately, the problem with TDE is that it is impossible to produce a finite length CES that has both a low PAPR and an ideal ACF. This means that the ACF sidelobes will lead to higher MSE in the channel estimate, with the hope being that the MMSE can compensate.

In this paper, we combine FDE and TDE to develop a hybrid CES that permits the receiver to select the appropriate method 


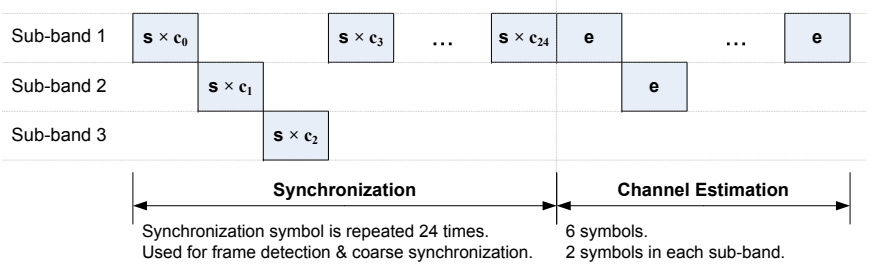

Fig. 2. MB-OFDM preamble.

based on channel conditions and available computational resources. With both the CES and the packet preamble based on a complementary sequence pair, we will show how both flexibility and performance can be increased. In other words, this approach allows for heterogenous receiver architectures wherein a sophisticated receiver can obtain superior performance while a low-cost receiver can focus on minimizing its signal processing overheads.

Although this work is relevant to many OFDM systems, including IEEE 802.11a and 802.11g, we focus on MBOFDM, the first ultra-wideband (UWB) technology to obtain international standardization [5]. MB-OFDM is representative of contemporary OFDM systems in that it supports several data rates. With short impulsive UWB channels suffering almost entirely from AWGN and long UWB channels susceptible to severe frequency-selective fading, it is critical that channel estimation be robust, accurate and low-complexity in all cases. Since different UWB devices will place performance or complexity as paramount, MB-OFDM is ideal context in which to evaluate a flexible receiver-based algorithm.

We begin by detailing the motivation for hybrid CES in Section II. Then, in Section III, we consider the metrics by which we can obtain an optimal set of complementary sequence pairs for synchronization and channel estimation. Monte Carlo simulations are then used in Section IV to quantify the end-to-end packet error rates (PERs) and give an example of several high-performing preamble sequences for MB-OFDM. Finally, we present our conclusions in Section $\mathrm{V}$.

\section{Motivation}

The MB-OFDM packet header, which is representative of all OFDM systems, is shown in Fig. 2 We see that there are two distinct phases to the packet preamble. The packet begins with a $N=128$ chip synchronization sequence (SS) $\mathbf{s}$ that is used to detect the presence of the packet on the channel and to determine frame timing. For example, in MB-OFDM, the SS is repeated 8 times in each of the three sub-bands. The CES e follows the SS and is used to estimate the frequency response of the channel.

As the receiver is not immediately aware when a transmission begins, the received SS can be denoted as

$$
r\left(n-n_{0}\right)=\sum_{k=0}^{L-1} t(n-k) h(k)+z(n)
$$

where $t(n)$ is the transmitted signal, $h(k)$ is a CIR of length $L, n_{0}$ is an unknown timing offset and $z(n)$ is AWGN with variance $\eta^{2}$. It is the role of the receiver synchronization process to resolve $n_{0}$ before the CES starts. If we assume that a given sequence has an ideal impulse auto-correlation

$$
\phi_{t t}(m)=\sum_{n=0}^{127} t(n+m) t(n)=\delta(m)
$$

then the cross-correlation between the transmitted and received signals will be

$$
\phi_{r t}\left(m-n_{0}\right)=\sum_{n=0}^{N-1} r\left(n+m-n_{0}\right) t(n)=\hat{h}\left(m-n_{0}\right)
$$

where $\hat{h}(n)$ is the estimated CIR with the only error due to AWGN.

The timing offset $n_{0}$ is resolved by finding the time at which the energy in a multipath capture window of $L$ samples is maximized. In other words, the metric

$$
p(m)=\sum_{i=0}^{L-1}\left|\phi_{r t}\left(m-n_{0}+i\right)\right|
$$

will be maximized at $n_{0}$.

From (3) we observe that, as well as resolving $n_{0}$, an ideal impulse SS will also yield an optimal estimate of the CIR without any computation beyond that needed for synchronization. This means that an ideal SS would make the CES redundant since the SS is repeated 8 times per subband, as opposed to only 2 times for the CES, the AWGN present in a TDE of the CIR could be up to $9 \mathrm{~dB}$ less than that of FDE. Further, with MMSE estimation incurring only only $N$ multiplications in the time-domain, TDE offers highperformance at low complexity.

Unfortunately, it is impossible to achieve the ideal impulse ACF assumed in (3) since the peak-to-average power (PAPR) must be as low as possible.

Further degrading the ACF is the fact that the frequency response of the SS must be as flat as possible in order to avoid incurring a reduction in transmit power to meet the spectral mask. For example, in the case of MB-OFDM, this is achieved by flattening the original frequency signal $\mathrm{s}$ via

$$
\begin{array}{r}
\mathbf{S}=F F T(\mathbf{s}) \\
\mathbf{S}_{n}^{\prime}=\frac{\mathbf{S}_{n}}{\left|\mathbf{S}_{n}\right|} \\
\mathbf{s}^{\prime}=\operatorname{IFFT}\left(\mathbf{S}^{\prime}\right) .
\end{array}
$$

We conclude that any practical SS will possess significant ACF sidelobes relative to the ideal ACF of (2) that will degrade the channel estimate of (3). In some cases, these sidelobes may even be so large that they make the SS unsuitable for use in channel estimation. For example, in MB-OFDM, the SS ACF sidelobes can exceed $-9.5 \mathrm{~dB}$ and will therefore quickly dominate AWGN.

The first motivation of this paper is therefore to find a new SS set that provides lower ACF sidelobes while retaining a low PAPR and flat PSD. This would allow a single 
preamble to support both TDE and FDE. In other words, a low-complexity receiver could use conventional FDE while a higher performance fast-synchronization receiver could utilize superior MMSE TDE.

The second motivation is to define the CES such that it forms a complementary sequence pair [6] with the SS. In other words, even though it is impossible to achieve an impulse ACF with a single finite-length bipolar sequence, it is possible to define a pair of such sequences that produce an impulse when their ACFs are added. This is a new approach to SS selection since previous techniques, such as evolutionary algorithms (EAs) [7], focus on optimizing the ACF of a single sequence [8].

In the next section, we will detail how a suitable complementary sequence pair for use as a SS and CES is obtained.

\section{Complementary Sequences for Channel ESTIMATION}

As stated previously, the optimal sequence for TDE possesses an impulse ACF. Although this is impossible for a finite-length binary sequence, it can be achieved with a complementary sequence pair. In other words,

$$
\phi_{s_{1} s_{1}}(n)+\phi_{s_{2} s_{2}}(n)=2 \delta(n)
$$

where $s_{1}(n)$ and $s_{2}(n)$ denote pair of length $N$ complementary sequences. As shown in [9], complementary sequence pairs of length $N$ can be easily obtained via Golay-paired Hadamard matrices of order $K=\log _{2} N$. We can denote such a Golay-paired Hadamard matrix via the closed-form expression

$$
\mathbf{H}_{2^{K}}(i, j)=(-1)^{\Gamma}
$$

with

$$
\Gamma=\sum_{r=0}^{n-2}\left(j_{r+1} \oplus i_{r}\right) j_{r} \oplus i_{K-1} j_{K-1}
$$

where $j_{r}$ denotes the $r^{\text {th }}$ bit in the radix-2 expression ${ }^{1}$ of the column index $j$ as per

$$
j \equiv\left(j_{K-1}, j_{K-2}, \ldots, j_{0}\right)_{2}=\sum_{r=0}^{K-1} 2^{r} j_{r}
$$

and likewise for the row index $i_{r}$. We observe that $\mathbf{H}_{\left(2^{K}\right)}$ is an $N \times N$ matrix with the sequence denoted by row $k$ forming a complementary sequence pair with the sequence denoted by row $k+\frac{N}{2}$ where $0 \leq k<N / 2$. Further, by virtue of being based on the Hadamard matrix, each row $0 \leq k<N / 2$ will be orthogonal to every other row $0 \leq k<N / 2$. This latter property is critical for MB-OFDM wherein SS orthogonality is used to distinguish between piconets.

In the context of the preamble of Fig. 2, we therefore propose that the sequence $s_{1}$ be used as the SS and the sequence $s_{2}$ be used as the CES. Although this means that the synchronization will continue to suffer from a non-ideal ACF, the TDE will be able to fully exploit the complementary nature of the sequences with nominal complexity.

\footnotetext{
${ }^{1}$ All radix-2 notations are written MSB to LSB
}

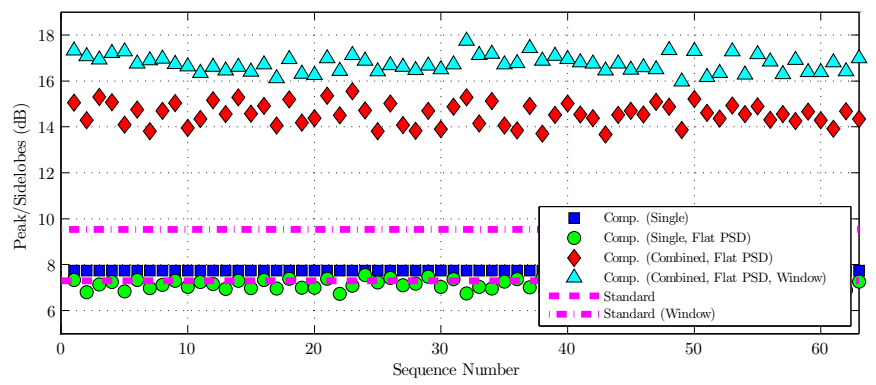

Fig. 3. Relative magnitude of ACF sidelobes.

Although the bipolar complementary sequences could be used as-is, it may be beneficial to flatten the PSD of both sequences as discussed in Section II. While this means that the sequences will no longer be perfectly complementary, thereby increasing ACF sidelobes, such losses will be offset by the reduction in transmit power back-off.

Further, when the PSD of the CES is flattened, the resulting sequence can be used for a FDE of the CIR in the same way as a conventional QPSK-based sequence. Furthermore, even though a conventional FDE based on QPSK constellations can use complex conjugation to avoid multiplication, there is no real hardware saving since a complex multiplication will still be necessary during equalization. We can therefore conclude that any sequence with a flat PSD will provide equivalent performance in terms of FDE potential.

In the context of MB-OFDM, where $N=128$, we observe that (7) will produce a pool of 64 complementary sequence pairs. Since MB-OFDM uses the SS to distinguish between up to 7 simultaneous piconets, we can discard the 57 worstperforming sequences. To that end, Fig. 3 shows the relative cumulative magnitude of the autocorrelation sidelobes for all complementary sequences of length $N=128$. Note that rather than consider all ACF sidelobes, we can utilize a window that considers only the first 32 sidelobe offsets since the signal can only recover multipath delays within the 32 sample zero pad (ZP) regardless of the quality of the channel estimate.

We observe from Fig. 3 that the SS sequence used in the current MB-OFDM standard has sidelobes $7.5 \mathrm{~dB}$ below the ACF peak. When $s_{1}$ of the best complementary pair is considered on its own, we see that it has a marginal improvement. When the PSD is flattened, the sidelobes increase by approximately $1 \mathrm{~dB}$ on average. The contribution of the PSD flattening to the sidelobes can be reduced by over 8 $\mathrm{dB}$ when the complementary sequences are combined. From this analysis, we conclude that the sequence denoted by row $k=23$ of (7) is optimal. Furthermore, sequences 21, 3, 14, 32,50 and 18 would also be recommended for use for the other MB-OFDM time-frequency codes (TFCs).

Another perspective on the difference between sequences is shown in Fig. 4. Here, the ACF of the standard SS is compared to the flattened optimal sequence, both individually and when combined with its complement. We observe that the standard SS has four significant sidelobes within the first 20 offsets 


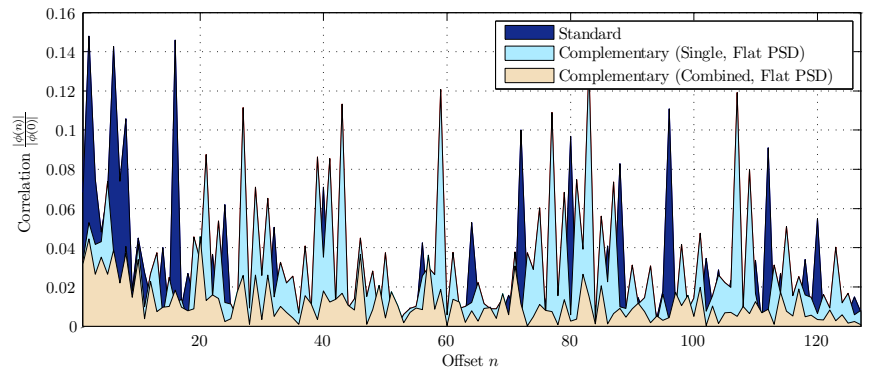

Fig. 4. Comparison of auto-correlation sidelobes for reference and complementary sequences.

of the correlation peak that will cause significant distortion if the sequence were to be used for TDE. Although the complementary sequence also has significant sidelobes when considered in isolation, the fact that they do not occur within the ZP window greatly reduces their significance. Finally, we can again see that the combined complementary sequence pair is clearly superior even when its PSD is flattened.

We have observed that the ACF of one-half of a wellchosen complementary pair is comparable to the existing MBOFDM SS. Further, when a complementary pair is combined, the ACF becomes ideal. We therefore conclude that a PSDflattened complementary-sequence-based SS and CES will allow for superior performance and improved flexibility since the decision between FDE and TDE can be made at the receiver.

\section{Synchronization PeRformance}

In this section, we model how the selection of the SS impacts on the synchronization performance of the receiver. Since the goal of synchronization is to find a window of $L$ samples, with $L=32$ in the case of MB-OFDM, wherein the most multipath signal components can be recovered, we will measure performance as being the ratio of the signal energy within the window to the total signal energy. For example, in a multipath channel that had equal magnitude signal components arriving at delays of 0,20 and 40 samples, the best possible result for an $L=32$ receiver would be a capture efficiency of $\frac{2}{3}(-1.7 \mathrm{~dB})$.

To ensure that our simulation uses realistic channel conditions, we will utilize the 4 UWB channel models defined by the IEEE 802.15.3a working group [10]. Denoted as CM1 through CM4, these models consider both line-of-sight (LOS) and non-line-of-sight (NLOS) conditions and possess average RMS delay spreads of $5 \mathrm{~ns}$ to $25 \mathrm{~ns}$. In these simulations, we will focus on the highly-dispersive CM4 channel since it is representative of worst-case situations.

Fig. 5 shows the average energy lost due to imperfect synchronization at a range of SNRs. To establish a baseline reference, the ideal response disregards PAPR considerations and uses an impulse sequence. We can observe that the SS specified by the MB-OFDM standard is inferior to the ideal, particularly at low SNRs. Interestingly, when one-half the optimal complementary sequence pair identified in the

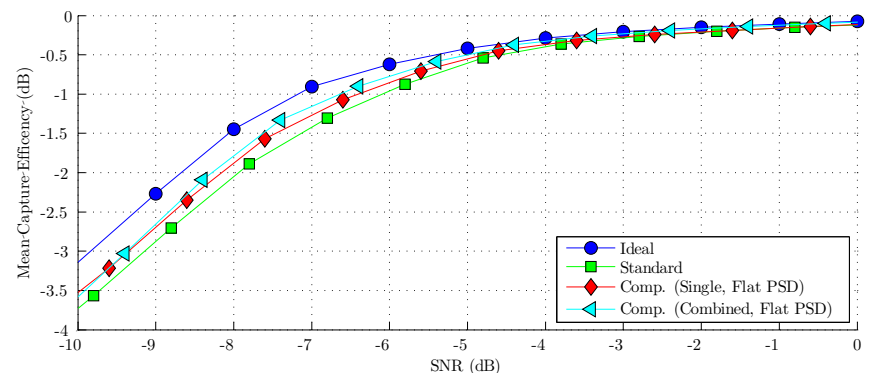

Fig. 5. Capacity efficiency vs. SNR

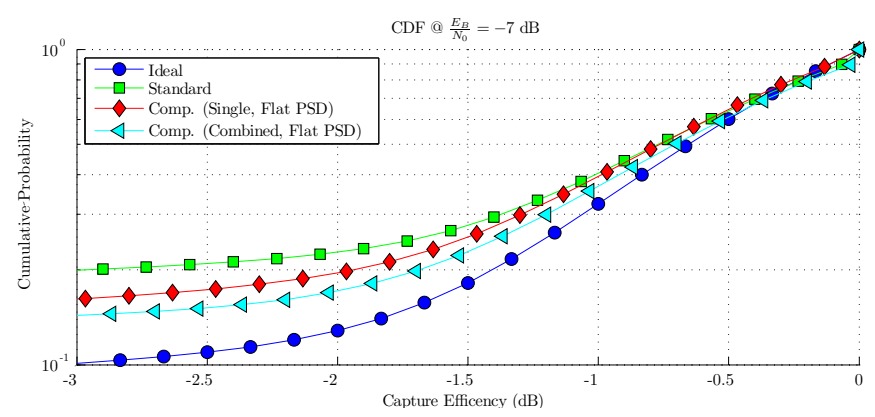

Fig. 6. Cumulative distribution of capture efficiency at $\frac{E_{B}}{N_{0}}=-7 \mathrm{~dB}$.

previous section is used, performance marginally improves. Then, when this sequence is combined with its complement, an additional increase in performance is noted. Unfortunately, since the synchronization is only aware of one sequence, as per Fig. 2, the combined response is has no real practical application.

Another perspective on synchronization performance is shown in Fig. 6, where we use the cumulative distribution of the previous data set to consider the probability of the capture efficiency being worse than an arbitrary threshold. We observe that, at an $\frac{E_{B}}{N_{0}}$ of $-7 \mathrm{~dB}$, an ideal SS has a $10 \%$ probability of having a capture efficiency less than $3 \mathrm{~dB}$. Conversely, when the standard SS is used, this probability increase to $20 \%$. The performance of the complementary sequences fall in-between these two extremes.

We have observed that the SS recommended by the MBOFDM standard is inferior to the complementary sequences identified in the previous section. We therefore conclude that there will be no degradation to synchronization performance by using complementary sequences for flexible timedomain/frequency-domain channel estimation.

\section{Channel Estimation}

With it demonstrated that a complementary sequence-based preamble incurs no loss during synchronization, we now consider the performance of channel estimation. Accordingly, this section uses Monte Carlo simulations to compare both TDE and FDE for different preambles.

\section{A. Mean Squared Error}

Fig. 7 shows the MSE for LS and MMSE channel estimations when the power of all estimation sequences is constant. 

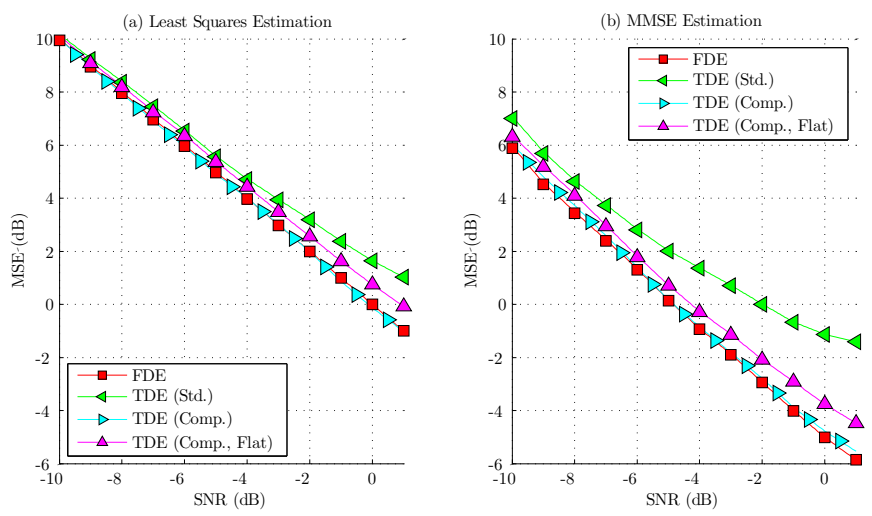

Fig. 7. MSE of channel estimate with equal power sequences.

In other words, differences in performance are due solely to the quality of the CES. We observe from Fig. 7a that the MSE when using FDE is equivalent to the magnitude of the AWGN. This is also equivalent to a TDE based on an unmodified bipolar complementary sequence, which is to expected given that the complementary sequence yields a perfect estimate of the CIR. When we flatten the frequency response of the complementary sequence, as per (5-6), we observe some degradation in low-noise conditions. We note that these losses are always less than a TDE based on the standard MB-OFDM SS. We therefore conclude that least squares (LS) estimation should always be done via FDE since TDE offers no savings in computational efficiency to justify its performance loss.

In short channels, as argued in Section II, MMSE estimation offers significant advantages since it exploits sub-carrier correlations to reduce the impact of AWGN. This is shown in Fig. 7b. Although we observe a reduction in MSE in all cases, we note that the poor ACF of the standard SS becomes very significant while the flattened complementary sequence remains within $1 \mathrm{~dB}$ of the ideal FDE.

With FDE possessing the best performance when the $\frac{E_{B}}{N_{0}}$ is kept constant, we now consider the impact of placing the SS and CES in the MB-OFDM preamble. For example, with Fig. 2 showing the SS repeated 8 times per sub-band and the CES repeated twice per sub-band, it is apparent that utilizing the SS for estimation will improve performance by virtue of reducing the AWGN. Note that, in these simulations, we will assume 6 repetitions of the SS are available for TDE since the first two are assumed to be lost due to packet detection, automatic gain control (AGC) calibration and synchronization.

Fig. 8 shows the MSE when one takes advantage of the fact that sequences are repeated several times in the packet preamble, as per Fig. 2. In other words, the FDE sequences are average over two repetitions, which occur during the channel estimation part of the preamble, whereas the TDE sequences can also be averaged over the synchronization part of the preamble, giving a total of ten repetitions. Although this would nominally reduce the impact of AWGN on TDE relative to FDE by a factor of 5 , we would expect that the noise-floor from the imperfect ACF will impose a practical
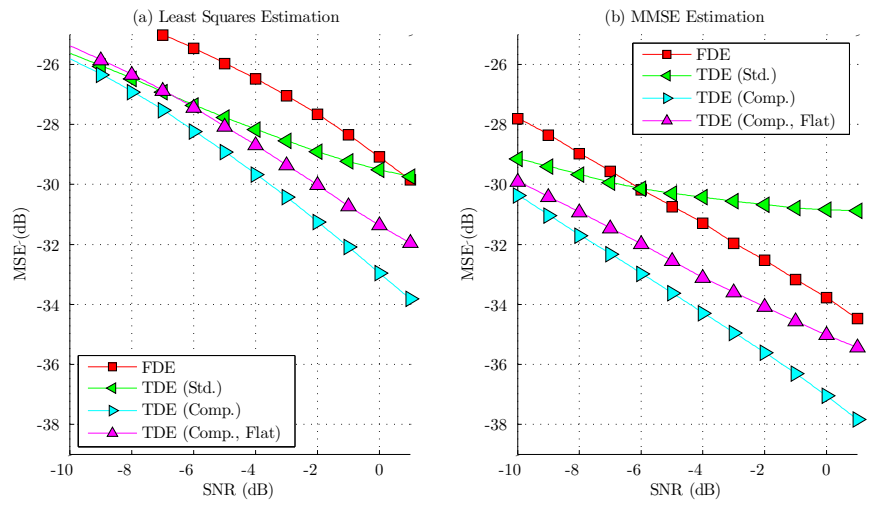

Fig. 8. MSE of channel estimate using preamble format of Fig. 2.

limit on the gains. From the figure, we can observe that FDE, using only the two repetitions of the CES, has the worst performance at low SNR. However, as the AWGN becomes less significant, the poor ACF of the standard MB-OFDM SS dominates the reduction in AWGN and thereby TDE losses its performance advantage. This is particularly obvious when MMSE estimation is used as in Fig. 8(b). Our overall conclusion is therefore that a complementary sequence-based TDE can offer significant advantages in poor channels with a low SNR.

It is important to note that Fig. 7 and 8 denote extreme cases and are dependant on the number of sequence repetitions in the preamble. In other words, for low-complexity receivers that require all eight repetitions of the SS for synchronization, the only reason to use a TDE based on a complementary sequence is to reduce the complexity of MMSE estimation. However, for higher-complexity devices, TDE can reduce estimation error by several $\mathrm{dB}$.

It is apparent that a complementary-sequence based preamble that enables both TDE and FDE offers the potential for higher performance at lower complexity. Although not all receivers may be able to take advantage of such a SS, we note that the worst-case result, with FDE performed via the CES alone, would be equivalent to that of the current MB-OFDM preamble used in conventional FDE.

\section{B. Packet Error Rates}

In this section we present the end-to-end performance in terms of packet error rate (PER). A reference is provided in terms of the ideal PER, which is obtained by assuming a perfect equalizer, i.e. the only loss is due to AWGN in the packet payload. In all cases, we assume MMSE estimation is used.

We begin by considering the high-noise case of Fig. 9 . This simulation was obtained using the $53.3 \mathrm{Mb} / \mathrm{s}$ data rate of the MB-OFDM standard, which has a combined process and coding gain of $\frac{53.3}{512} \approx 10 \mathrm{~dB}$. We see that under highnoise conditions, the reduction in AWGN achieved by TDE is so significant that the PER improves even when the SS is the relatively poor sequence used in standard MB-OFDM. 


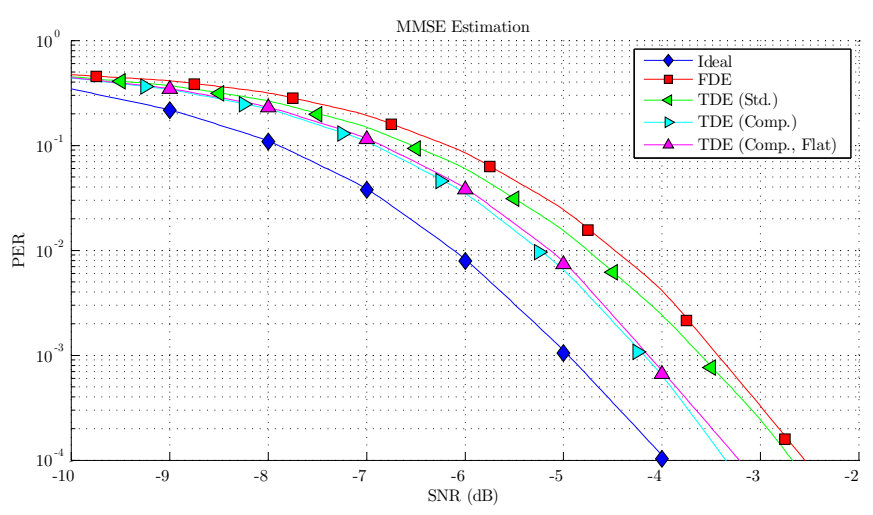

Fig. 9. PER for difference channel estimation techniques.

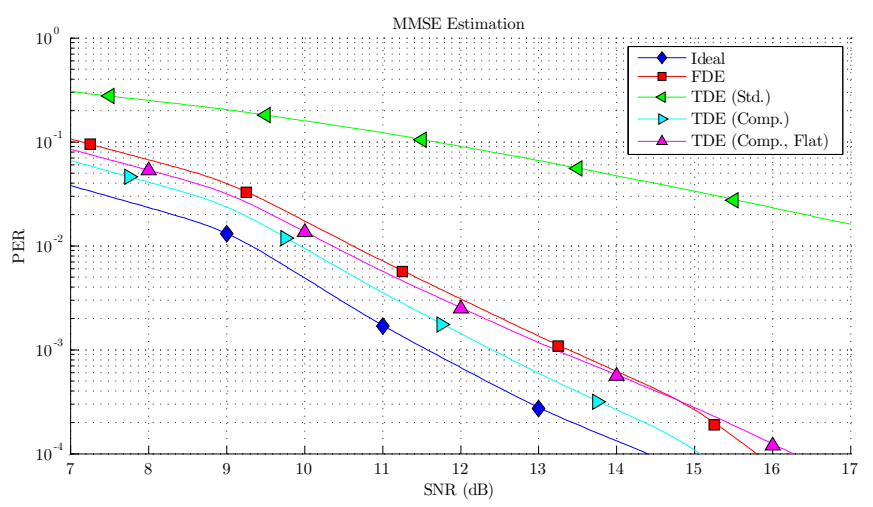

Fig. 10. PER for difference channel estimation techniques.

We also observe that the difference between the original and flattened complementary sequences is negligible; both approaches improve performance by approximately $0.6 \mathrm{~dB}$.

The results under low-noise conditions, as shown in Fig. 10 , are very different. With the lower AWGN allowing for a higher data rate, this simulation was conducted at $480 \mathrm{Mb} / \mathrm{s}$, where the combined process and coding gain is only 0.28 $\mathrm{dB}$. We can now observe that it is critical that TDE use a sequence with low ACF sidelobes; the MB-OFDM standard SS leads to performance losses exceeding $6 \mathrm{~dB}$. Even the smaller ACF sidelobes belonging to flattened complementary sequence have become so significant that there is no longer a performance advantage over FDE (although the computational savings remain).

We therefore conclude that the best PER can be efficiently achieved by designing a receiver that uses TDE for low-rate high-SNR communications and FDE for high-rate low-SNR communications. This means that a complementary-sequence based packet preamble is attractive since it allows a single transmitted signal to support multiple receiver architectures.

\section{CONCLUSION}

We began by identifying the relative advantages of FDE and TDE for OFDM systems, namely that FDE can offer low-complexity LS channel estimation while TDE offers lowcomplexity MMSE channel estimation. With the best channel estimation method dependant on receiver architecture and channel conditions, we have argued for a new type of CES that possesses a flat PSD, which allows FDE to be used, as well as an impulsive ACF, which enables TDE as well.

Since it is impossible to obtain a single finite-length sequence that meets these criteria, we have designed a new type of packet preamble wherein the SS and CES are based on a complementary sequence pair. A comparison with the conventional SS used in the UWB MB-OFDM standard has revealed that the synchronization performance of the new scheme is almost unchanged. However, in terms of channel estimation MSE and end-to-end PER, the new scheme offers significant advantages.

Our overall conclusion is that a complementary sequencebased preamble allows for increased performance and better computational efficiency in sophisticated receivers without impact to low-cost alternatives.

\section{REFERENCES}

[1] Z. Wang and G. B. Giannakis, "Wireless multicarrier communications," IEEE Signal Processing Magazine, vol. 17, no. 3, pp. 29-48, 2000.

[2] M. Luise, R. Reggiannini, and G. M. Vitetta, "Blind equalization/detection for OFDM signals over frequency-selective channels," IEEE Journal on Selected Areas in Communications, vol. 16, no. 8, pp. $1568-1578$, Oct. 1998.

[3] A. Batra, J. Balakrishnan, G. R. Aiello, J. R. Foerster, and A. Dabak, "Design of a multiband OFDM system for realistic UWB channel environments," in IEEE Transactions on Microwave Theory and Techniques, vol. 52, no. 9, Sept. 2004, pp. 2123-2138.

[4] J. J. van de Beek, O. Edfors, M. Sandell, S. K. Wilson, and P. O. Brjesson, "On channel estimation in OFDM systems," in Proceedings of the IEEE Vehicular Technology Conference, July 1995, pp. 815-819.

[5] High Rate Ultra Wideband PHY and MAC Standard, ECMA International ECMA-368, Dec. 2005.

[6] M. Golay, "Sieves for low autocorrelation binary sequences," IEEE Transactions on Information Theory, vol. 23, no. 1, pp. 43-51, Jan. 1997.

[7] H. Dong, B. Wang, C. Gu, and G. Feng, "Synthesis of binary sequences with good auto- and cross-correlation properties by GA," in Proceedings of International Conference on Communication Technology, Oct. 1998, p. 5.

[8] O. Ureten, S. Tascioglu, N. Serinken, and M. Yilmaz, "Search for OFDM synchronization waveforms with good aperiodic autocorrelations," in Proceedings of CCECE, May 2004, pp. 13-18.

[9] X. Huang and Y. Li, "Scalable complete complementary sets of sequences," in GLOBECOM 2002, Taipei, Taiwan, Nov 2002.

[10] A. F. Molisch, J. R. Foerester, and M. Pendergrass, "Channel models for ultrawideband personal area networks," IEEE Wireless Commun. Mag., pp. 14-21, Dec. 2003. 